\title{
OPTIMAL RELAY DESIGN OF ZERO FORCING EQUALIZATION FOR MIMO MULTI WIRELESS RELAYING NETWORKS
}

\author{
Apriana Toding \\ Dept. Electrical Engineering \\ Universitas Kristen Indonesia Paulus Makassar, Indonesia \\ E-mail: apriana.toding@ukipaulus.ac.id
}

\begin{abstract}
In this paper, we develop the optimal relay design for multiple-input multiple-output (MIMO) multi wireless relaying networks, when we consider the problem of zero-forcing processing is studied for multi-input multi- output multi-relay communication system in which MIMO source-destination pairs communicate simultaneously. It is assumed that due to severe shadowing effects which communication links can be established only with the aid of relay node. The aim is to design the relay amplification matrix to maximize the achievable communication sum-rate through the relay, which in general amplifyingand-forward relaying mechanisms are considered. The zero forcing $(Z F)$ algorithm has studied for a MIMO multi relay network by comparing its performance in terms of bit- error-rate (BER) at destination algorithm. In particular, we investigate its performance with and without using the $Z F$ at the relay. Our results demonstrate that the system performance can be significantly improved by using the ZF algorithm at relay (optimal relay $Z F$ algorithm).
\end{abstract}

Keywords: multiple-input multiple-output (MIMO), multi-relay networks, zero-forcing (ZF), bit-error-rate. 


\section{INTRODUCTION}

In order to provide a reliable wireless transmission, one needs to compensate for the effects of signal fading due to multi-path propagation and strong shadowing. One way to address these issues is to transmit the signal through one relay [1]-[10] or more relays [11], [12], which can be accomplished via a wireless network consisting of geographically separated nodes. And then the basic motivation behind the use of cooperative communications lies in the exploitation of spatial diversity provided by the network nodes [5], [8], [11], [12], as well as the efficient use of power resources [5]-[11] which can be achieved by a scheme that simply receives and forwards a given information, yet designed under certain optimality criterion.

Relay schemes can be broadly categorized into three general groups: decode-and-forward (DF), compress-and-forward (CF), and amplify-and-forward (AF). In the DF scheme, the relay nodes first decode the received signals and then forward the re-encoded signals toward the destination node [1]. In the CF scheme, the relay nodes compress the received signals by exploiting the statistical dependencies between the signals at the nodes [3]. In the AF scheme, the relay nodes amplify the received signal and rebroadcast the amplified signals toward the destination node [4]. In this paper we consider the AF strategy which is easier to implement compared with the other two approaches.

When there is one relay node between source- destination multiple-input multipleoutput (MIMO), we call such system MIMO relay communication system [1]-[10]. Also when there are more relay node between source destination MIMO, we call such system MIMO multi-relay communication system [11], [12]. Recently, MIMO relay and multi-relay communication systems have attracted much research interest and provided significant improvement in terms of both spatial efficiency and link reliability. In this paper, we investigated the performance of optimal relay zero-forcing (ZF) algorithm in a MIMO multirelay communication system in terms of biterror-rate (BER) that are not included in [11], [12]. Note that the ZF algorithm has already been studied with MIMO relay communication system [2] and [4]. In this paper, we study the optimal relay ZF algorithm in MIMO multi- relay communication system. Our results show that algorithms in MIMO multi-relay communication system have significant performance improvement.

The rest of the paper is organized as follows: the system model is described in Section System Model for MIMO Multy-Relay; in Section Optimal Relay ZF, we study the optimal relay $\mathrm{ZF}$ in a MIMO multi-relay communication system; Section Result and Discussion shows the simulation results with ZF, MMSE and optimal relay ZF algorithms under various system scenarios and the conclusion is given in Conclusion Section.

\section{SYSTEM MODEL FOR MIMO MULTY-RELAY}

Figure 1 illustrates a two-hop MIMO relay communication system consisting of one source node, $K$ parallel relay nodes, and one destination node. We assume that the source and destination nodes have $N_{s}$ and $N_{d}$ antennas, respectively, and each relay node has $N_{r}$ antennas. The generalization to the system with different number of antennas at each relay node is straightforward. To efficiently exploit the system hardware, each relay node uses the same antennas to transmit and receive signals. Due to its merit of simplicity, we consider the amplify- and-forward relaying scheme at each relay. The communication process between the source and destination nodes is completed in two time slots.

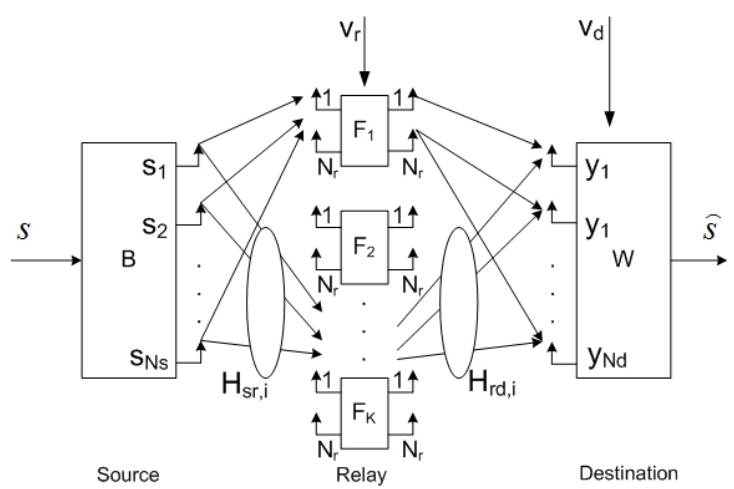

Figure 1: System model for MIMO MultiRelay Channel 
In the first time slot, the $N_{b} \times 1$ modulated symbol vectors is linearly precoded as

$x=B s$

Where, $B$ is an $N_{s} \times N_{b}$ source precoding matrix. We assume that $E\left[s s^{H}\right]=I_{N}$, where $(.)^{H}$ denotes matrix (vector) Hermitian transpose, $E[$.$] stands for statistical expectation, and In is$ an $n \times n$ identity matrix. The precoded vector $x$ is transmitted to the relay nodes and the received signal at the ith relay node can be written as

$y_{r, i}=H_{s r, i} x+v_{r, i}, \quad i=1, \ldots, K$

where $H_{s r, i}$ is the $N_{r} \times N_{s}$ MIMO channel matrix between the source and the $i^{\text {th }}$ relay node, $y_{r, i}$ and $v_{r, i}$ are the received signal and the additive Gaussian noise vectors at the $i^{\text {th }}$ relay node, respectively.

In the second time slot, the source node is silent, while each relay node transmits the amplified signal vector to the destination node as

$x_{r, i}=F_{i} y_{r, i}, \quad i=1, \ldots, K$

where $\mathrm{Fi}$ is the $\mathrm{Nr} \times \mathrm{Nr}$ amplifying matrix at the ith relay node. Thus the received signal vector at the destination node can be written as

$y_{d}=\sum_{i=1}^{K} H_{r d, i} x_{r, i}+v_{d}$

where $\mathrm{H}_{\mathrm{rd}, \mathrm{i}}$ is the $\mathrm{N}_{\mathrm{d}} \times \mathrm{N}_{\mathrm{r}}$ MIMO channel matrix between the ith relay and the destination node, $\mathrm{y}_{\mathrm{d}}$ and $\mathrm{v}_{\mathrm{d}}$ are the total received signal and the additive Gaussian noise vectors at the destination node, respectively.

Substituting (1)-(3) into (4), we obtain

$$
\begin{aligned}
y_{d} & =\sum_{i=1}^{K}\left(H_{r d, i} F_{i} H_{s r, i} B s+H_{r d, i} F_{i} v_{r, i}\right)+v_{d} \\
& =H_{r d} F H_{s r} B s+H_{r d} F v_{r}+v_{d}
\end{aligned}
$$

where we define

$$
\begin{aligned}
H_{s r} & \triangleq\left[\left(H_{s r, 1}\right)^{T},\left(H_{s r, 2}\right)^{T}, \ldots,\left(H_{s r, K}\right)^{T}\right]^{T} \\
H_{r d} & \triangleq\left[H_{r d, 1}, H_{r d, 2}, \ldots, H_{r d, K}\right] \\
F & \triangleq b d\left[F_{1}, F_{2}, \ldots, F_{K}\right] \\
v_{r} & \triangleq\left[\left(v_{r, 1}\right)^{T},\left(v_{r, 2}\right)^{T}, \ldots,\left(v_{r, K}\right)^{T}\right]^{T}
\end{aligned}
$$

Here $(.)^{\mathrm{T}}$ denotes the matrix (vector) transpose, bd(.) stands for a block-diagonal matrix, $H_{s r}$ is a $K N_{r} \times N_{s}$ channel matrix between the source node and all relay nodes, $H_{r d}$ is an $N_{d} \times K N_{r}$ channel matrix between all relay nodes and the destination node, $v_{r}$ is obtained by stacking the noise vectors at all the relays and $F$ is the equivalent $K \mathrm{Nr} \times \mathrm{KNr}$ block diagonal relay matrix. The diagram of the equivalent MIMO relay system described by (5) is shown in Figure 2.

By introducing

$\bar{F} \triangleq H_{r d} F$

the received signal vector at the destination can be equivalently written as

$y_{d}=\bar{F} H_{s r} B s+\bar{F} v_{r}+v_{d}=\bar{H} s+\bar{v}$

where we define $\bar{H} \triangleq \bar{F} H_{s r} B$ as the effective MIMO channel matrix of the source-relaydestination link, and $\mathrm{v}$ as the equivalent noise with $\overline{\boldsymbol{v}} \triangleq \bar{F} v_{r}+v_{d}$.

In this paper, we try to improve the system BER performance by using ZF equalizer. A simple approach to design the relay is to treat it as an all-pass AF unit, which we construct as $F=\alpha I_{N r}$, where $\alpha$ is the amplifying factor of the relay and $\mathrm{I}_{\mathrm{Nr}}$ is an identity are the transmit power available at the source and the matrix of dimension $\mathrm{N}_{\mathrm{r}}$. We can find $\alpha$ from $P r=\alpha^{2} \operatorname{tr}\left\{P s / N s\left(H_{S r} H_{S R}^{H}+I_{N r}\right\}\right.$. Here $\mathrm{Ps}>\mathrm{O}$ and $\mathrm{Pr}>0$ relay nodes respectively, $(.)^{\mathrm{H}}$ denotes matrix Hermitian and $\operatorname{tr}\{$.$\} indicates trace of a matrix.$

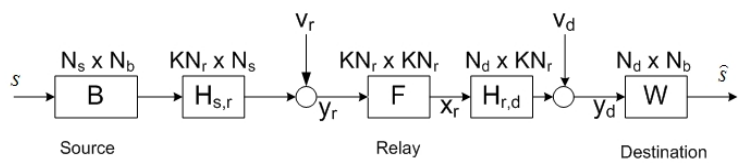

Figure 2 : Equivalent MIMO Relay Channel.

\section{OPTIMAL RELAY ZF IN MIMO MULTI-RELAY SYSTEM}

We study the following detection algorithms for MIMO multi-relay systems such as the ZF equalizer. If we consider the received signal vector at the destination in (5) then our proposed MIMO multi-relay channel (Figure 1) reduces to a MIMO relay channel (Figure 2) 
into MIMO channel with the equivalent channel matrix of $H s+\boldsymbol{v}$ where $H=\bar{F} H_{s r} B$, the signals vector of $\mathrm{s}$ and the equivalent noise vector of $v=\bar{F} v_{r}+v_{d}$. Now we can analyze the signal detection at the relay receiver with the zero-forcing equivalent MIMO channel.

The first decoding technique to be described in this paper is the performance zero-forcing (ZF) technique at relay (ZFR). The relay uses the channel estimate and $\mathrm{ZF}$ technique to combine the received signals from all source antennas as (2) at the relay

$y_{r}=H_{s r} x+v_{r}$

$\hat{\mathrm{y}}_{\mathrm{r}}=\mathrm{W}^{\mathrm{H}} \mathrm{y}_{\mathrm{r}}$

The $\mathrm{ZF}$ technique meeting the constraint $\mathrm{W}^{\mathrm{H}} \mathrm{H}=\mathrm{I}_{\mathrm{Ns}}$ is given by

$W_{r}=H_{s r}\left(H_{s r}^{H} H_{s r}\right)^{-1}$

$\mathrm{Wr}$ is also known as the pseudo-inverse for a general $\mathrm{N}_{\mathrm{r}} \times \mathrm{N}_{\mathrm{s}}$ matrix and (.) $)^{-1}$ indicates simple matrix inversion. In order for a pseudo-inverse to exist, $\mathrm{N}_{\mathrm{r}}$ must be greater than or equal to $\mathrm{N}_{\mathrm{s}}$. Then, we derived the performance zero-forcing (ZF) technique at destination (ZFD). The estimated signal at destination (5) is given by

$\hat{s}=W_{d}^{H} y_{d}$

$W_{d}=H\left(H^{H} H\right)^{-1}$

$\mathrm{W}_{\mathrm{d}}$ is also known as the pseudo-inverse for a general $N_{d} \times N_{s}$ matrix and $(\cdot)^{-1}$ indicates simple matrix inversion. In order for a pseudoinverse to exist, $N_{d}$ must be greater than or equal to $N_{s}$. Using the zeroforcing technique approach described above at the relay and destination.

\section{RESULT AND DISCUSSION}

In the simulations, the transmission signaling is in spatial multiplexing mode (i.e., the source transmits independent data streams from different antennas) with total transmit power uniformly distributed among the transmit antennas. We study the performance of the proposed zero-forcing technique algorithm for MIMO multi-relay systems. All simulations are conducted in a flat Rayleigh fading environment using the BPSK constellation, and the noises are i.i.d. Gaussian random variables with zero mean and unit variance. The channel matrices have zero-mean entries with variances $\sigma_{s}^{2} / N_{s}$ and $\sigma_{r}^{2} /(K)$ for $H_{s r}$ and $H_{r d}$ respectively. We transmitted $10^{3}$ randomly generated bits in each channel realization and the BER results are averaged through 200 channel realizations. We plot BER curves versus SNR.

In the first example, we simulate the system BER performance of ZF and MMSE at destination algorithms with optimal relay ZF at relay and destination algorithm (proposed algorithm) in MIMO multi relay channel with varying SNR in the source-to-relay link (SN $\mathrm{R}_{\mathrm{s}}$ ) keeping the relay-to-destination SNR (SN $\mathrm{R}_{\mathrm{r}}$ ) at $25 \mathrm{~dB}$. Figure 3 show the BER performance with $N_{s}=N_{r}=N_{d}=3$ with the effect of the number of relays $(K=2)$. It can been seen that at $\mathrm{BER}=10^{-2}$, we achieve $10 \mathrm{~dB}$ gain from $\mathrm{ZF}$ to optimal relay $\mathrm{ZF}$ algortihm.

In the second example, we study the effect of the number of relays to the system BER performance using the proposed algorithm. Figure 4 shows the BER performance with $K=2,4$, and 6 . It can be seen that at BER = $10^{-3}$, we achieve a $5 \mathrm{~dB}$ gain by increasing from $K=2$ to $K=5$.

In the third example, we simulate the system BER performance of ZF and MMSE at destination algorithms with optimal relay ZF at relay and destination algorithm (proposed algorithm) in MIMO multi relay channel with varying SNR in the relay-to-destination SNR $\left(\mathrm{SN} \mathrm{R}_{\mathrm{r}}\right)$ keeping the source-to-relay link (SN $\mathrm{R}_{\mathrm{s}}$ ) at $25 \mathrm{~dB}$. Figure 5 show the BER performance with $N_{s}=N_{r}=N_{d}=3$.

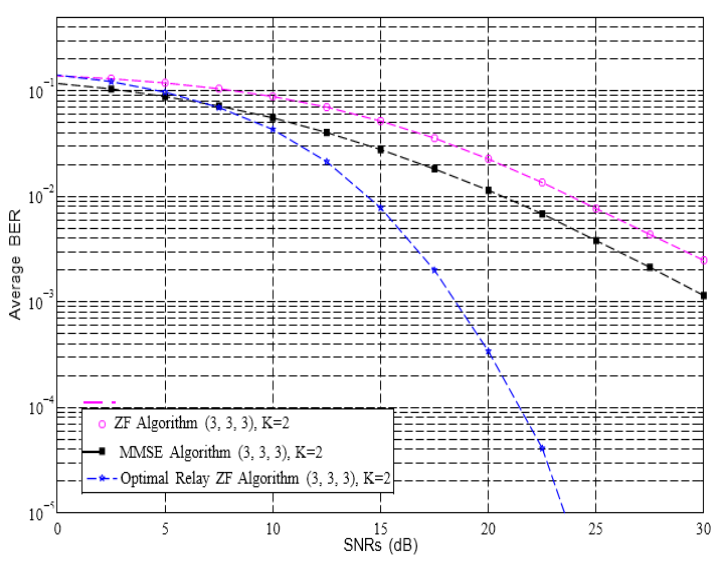

Figure 3: Example 1. BER versus $\mathrm{SN} \mathrm{R}_{\mathrm{s}} N_{s}=$ $N_{r}=N_{d}=3$ and SN $R_{r}=25 \mathrm{~dB}$ for MIMO Multi-Relay channel. 


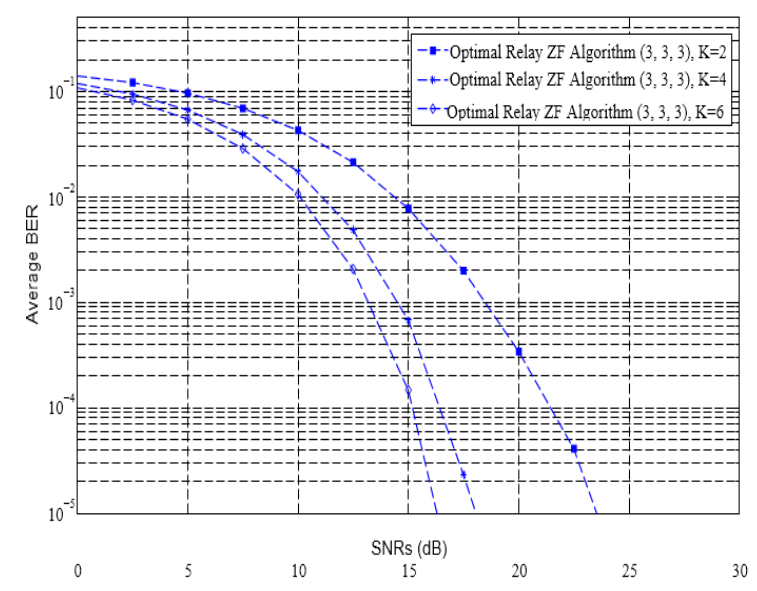

Figure 4: Example 2. BER versus $\mathrm{SN} \mathrm{R}_{\mathrm{s}} N_{s}=$ $N_{r}=N_{d}=3$ and SN $R_{r}=25 \mathrm{~dB}$ for MIMO Multi-Relay channel.

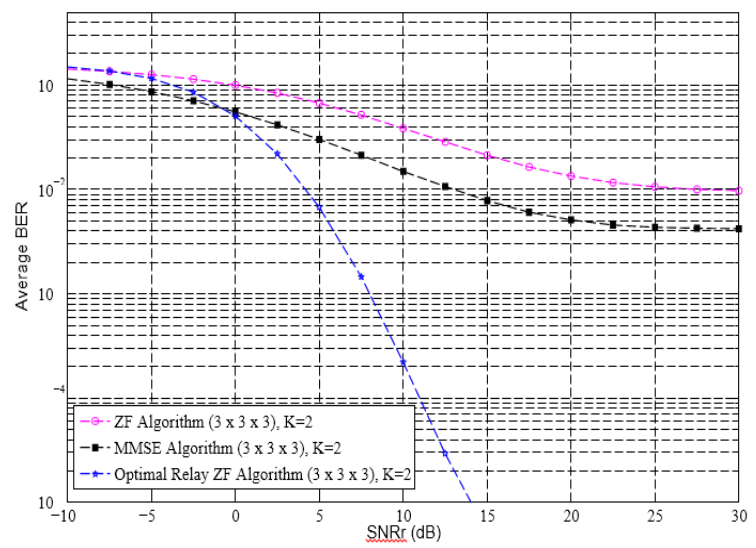

Figure 5: Example 3. BER versus $\mathrm{SN} \mathrm{R}_{\mathrm{s}} N_{s}=$ $N_{r}=N_{d}=3$ and SN $R_{r}=25 \mathrm{~dB}$ for MIMO Multi-Relay channel.

\section{REFERENCES}

[1] A. Kalantari and M. Neinavaie, "A diversity achieving power profile in MIMO decode and forward relay networks," in Information Technology, Electronics and Mobile Communication Conference (IEMCON), 2017 8th IEEE Annual, 2017, pp. 422-425.

[2] L. Gerdes, L. Weiland, and W. Utschick, "A zero-forcing partial decode-andforward scheme for the Gaussian MIMO relay channel," in Communications (ICC), 2013 IEEE International Conference on, 2013, pp. 3349-3354.
Our results demonstrate that optimal relay $\mathrm{ZF}$ algorithm has lower BER compared to the $\mathrm{ZF}$ and MMSE relay algorithms.

\section{CONCLUSION}

In conclusion, we have demonstrated the advantage of using ZF equalizer algorithm at relay in MIMO multi relay network by. We designed relays as all-pass amplify-andforward (AF) units which are simpler to implement. Our results demonstrate that optimal relay $\mathrm{ZF}$ algorithm at relay and destination outperform the ZF and MMSE algorithms at destination. Future works may include analysis the MMSE equalizer for MIMO multi-relay networks and optimizing the source and the relay matrices to allocate power efficiently in a cooperative MIMO relay network.

\section{ACKNOWLEDGMENT}

This work was supported under Ministry of Research, Technology, and Higher Education of Republic of Indonesia (Ristekdikti) and Universitas Kristen Indonesia Paulus Makassar (UKIP)

[3] S. Simoens, O. Muñoz-Medina, J. Vidal, and A. Del Coso, "Compress-and-forward cooperative MIMO relaying with full channel state information," IEEE Trans. Signal Process., vol. 58, no. 2, pp. 781791, 2010.

[4] A. Toding and Y. Rong, "Investigating successive interference cancellation in MIMO relay network," in TENCON 20112011 IEEE Region 10 Conference, 2011, pp. 359-363.

[5] W. Guan and H. Luo, "Joint MMSE transceiver design in non-regenerative MIMO relay systems," IEEE Commun. Lett., vol. 12, no. 7, 2008. 
[6] A. Toding and R. Arungla'bi, "Investigating Performance Zero-Forcing of Source Weighting Matrix in MIMO Relay Communication," in Prosiding International conference on Information Technology and Business (ICITB), 2015, pp. 9-13.

[7] A. S. Behbahani, R. Merched, and A. M. Eltawil, "Optimizations of a MIMO relay network," IEEE Trans. Signal Process., vol. 56, no. 10, pp. 5062-5073, 2008.

[8] Y. Rong, X. Tang, and Y. Hua, "A unified framework for optimizing linear nonregenerative multicarrier MIMO relay communication systems," IEEE Trans. Signal Process., vol. 57, no. 12, pp. 48374851, 2009.

[9] X. Tang and Y. Hua, "Optimal design of non-regenerative MIMO wireless relays,"
IEEE Trans. Wirel. Commun., vol. 6, no. 4, 2007.

[10]B. Wang, J. Zhang, and A. Host-Madsen, "On the capacity of MIMO relay channels," IEEE Trans. Inf. Theory, vol. 51, no. 1, pp. 29-43, 2005.

[11]A. Toding, M. R. Khandaker, and Y. Rong, "Joint source and relay design for MIMO multi-relay systems using projected gradient approach," EURASIP J. Wirel. Commun. Netw., vol. 2014, no. 1, p. 151, 2014.

[12]Apriana Toding and Syafruddin Syarif, "Performance Analysis of MIMO MultiRelay Networks With Zero-Forcing Equalizer," Proceeding Int. Conf. Ind. Technol. Sustain. Dev. 2017, Oct. 2017. 